\title{
Essais
}

ESSAIS

Revue interdisciplinaire d'Humanités

$8 \mid 2016$

Erreur et création

\section{La place du nom erreur et du verbe se tromper dans la langue française et la fonction heuristique de l'erreur dans la recherche}

Frédéric Lambert

\section{(2) OpenEdition \\ Journals \\ Édition électronique \\ URL : http://journals.openedition.org/essais/4897 \\ DOI : $10.4000 /$ essais. 4897 \\ ISSN : 2276-0970 \\ Éditeur \\ École doctorale Montaigne Humanités}

Édition imprimée

Date de publication : 15 mars 2016

Pagination : 18-25

ISBN : 978-2-9544269-7-6

ISSN : $2417-4211$

Référence électronique

Frédéric Lambert, «La place du nom erreur et du verbe se tromper dans la langue française et la fonction heuristique de l'erreur dans la recherche», Essais [En ligne], 8 | 2016, mis en ligne le 28 octobre 2020, consulté le 31 octobre 2020. URL : http://journals.openedition.org/essais/4897 ; DOI : https://doi.org/10.4000/essais.4897 


\section{La place du nom erreur et du verbe se tromper dans la langue française et la fonction heuristique de l'erreur dans la recherche}

\section{Frédéric Lambert}

Il y a deux erreurs sur l'erreur, l'une de la surestimer, l'autre de la sous-estimer'.

Edgar Morin

L'objet de cet article est de s'interroger, à partir de l'étude du couple que forment le nom erreur et le verbe se tromper en français, sur le concept qui les unit malgré leur divergence de forme. Les propriétés linguistiques et conceptuelles de ce couple permettent, à mon avis, d'envisager certains aspects de ce qu'on appelle communément la recherche sous un angle original. En effet, contrairement à beaucoup d'approches de l'erreur, qui en font un défaut dont il convient par tous les moyens de se garder, notre point de vue permet de reconsidérer la place de l'erreur dans la recherche et de ne pas y voir exclusivement une défaillance de l'esprit du chercheur aux prises avec l'inconnu. En ce qui concerne la sémantique lexicale, j'adopte ici une position cognitiviste au sens de George Lakoff', les mots renvoyant à des réseaux métaphoriques conceptuels.

Le nom erreur en français a cette particularité d'appartenir à ce qu'on pourrait appeler une famille recomposée, en ce sens que les termes qui partagent sa signification ont des formes phonétiques hétéroclites, alors que les mots proches morphologiquement ne partagent pas toujours le même sens. De ce point de vue, le français contemporain présente une situation de désordre qui contraste avec le latin ou même le français médiéval. Le verbe latin errare signifie à la fois " aller çà et là ", "se tromper de chemin ", "s'égarer " et "se tromper ". Parallèlement, et sans restriction, le nom error veut dire "action d'aller çà et là ", "incertitude ", " erreur ", " égarement " ou (en poésie) "tromperie ". Avec une autre image, le verbe labi "glisser ", " trébucher ", signifie aussi "se tromper " et a pour correspondant nominal lapsus, qui a pour significations " action de glisser, de trébucher ", " erreur ».

Edgar Morin, Enseigner à vivre, Paris, Actes Sud, 2014, p. 73.

2 George Lakoff, Metaphors We Live By, Chicago, University of Chicago Press, 2003. 
En italien, par exemple, errore n'a conservé que le sens d'" erreur " mais errare signifie à la fois «se tromper » et « aller à l'aventure ». Le lien sémantique entre les deux formes apparentées est donc en partie maintenu. C'est encore ce même lien qui associe error et errar en espagnol, ou erro et errar en portugais. En anglais, err est le correspondant verbal de error; et on sait que mistake est à la fois un nom et un verbe. En grec ancien, dans une autre orientation conceptuelle, au verbe hamartanein, qui signifie " manquer son but ", "se tromper ", s'associe le nom d'action hamartêma, et on a aussi, comme en latin, le verbe sphallesthai, qui signifie " trébucher » et « se tromper " qui a comme nom d'action «sphalma ».

Si on compare le français contemporain aux langues précédentes, on constate qu'il a accru la divergence sémantique entre le nom erreur et son correspondant verbal errer, dont le sens de "se tromper " est devenu littéraire. On notera que ce verbe ne doit pas être confondu avec le verbe errer (disparu au XVI e siècle) dont l'étymologie est le nom du voyage en latin, iter, gén. itineris, et dont il reste l'adjectif errant, qu'on trouve dans l'expression Juif errant. Mais, comme le note le $D H L F^{3}$, le verbe errer actuel «a été influencé par l'ancien verbe errer au point d'être confondu avec lui et de le remplacer ". Si on s'en tient donc au français courant actuel, le nom erreur n'a plus de lien sémantique (en apparence) avec le verbe errer et c'est le verbe pronominal se tromper qui lui correspond.

Tromper n'a pas une histoire très rectiligne non plus. En rapport avec la trompe et la trompette (issus du francique ${ }^{\circ}$ trumba), il aurait eu un emploi d'abord intransitif, au sens de " jouer de la trompe », puis un emploi pronominal figuré dans l'expression se tromper de quelqu'un, au sens de «se moquer de quelqu'un ", d'où viendrait ensuite l'emploi transitif au sens d'abuser ${ }^{4}$. Le verbe pronominal actuel s'interprète lui-même à partir de l'emploi transitif. Le verbe tromper, contrairement à erreur et errer, est assez riche en dérivés : trompeur, tromperie, détromper, trompe-l'œeil, trompe-la-mort. Mais on remarquera que tromperie ne peut pas servir de nominalisation au verbe pronominal correspondant : il n'a que le sens du transitif, ce qui explique que le mot erreur serve de substitut nominal du sens pronominal. On notera aussi que le pronominal se tromper n'est pas un transitif réfléchi comparable à se laver, qui peut se paraphraser par " laver soi-même ", mais plutôt un pronominal de sens passif, comme dans le cas de se tuer, qui a rarement le sens de "se suicider ", mais la plupart du temps celui d' "être tué " avec une valeur qu'on peut qualifier de progressive. Se tromper ne signifie donc pas «tromper soi-même ", mais plutôt "se retrouver en situation d'être trompé ».

3 Dictionnaire Historique de la langue française, Paris, Le Robert, 2012.

4 Le DHLG signale que Pierre Guiraud propose une autre étymologie, issue du latin triumphare, qui fait passer de "triompher » à " se moquer de " et enfin à " abuser ». 
Il y a ainsi une dissymétrie sémantique surprenante entre le verbe transitif tromper et le pronominal se tromper: alors que le premier implique une intention parfaitement consciente, qu' exprime clairement l'expression synonyme induire en erreur, le second est pratiquement incompatible avec le caractère intentionnel. Dire qu'on s'est volontairement trompé annule en fait une propriété essentielle de l'erreur, car ce n'est plus l'auteur de l'erreur qui est trompé mais un autre destinataire qui se trouve ainsi induit en erreur. Mais les questions actancielles ne se limitent pas à l'opposition actif/ pronominal. Quand le sujet de tromper à l'actif n'est pas un animé humain ou un équivalent, le caractère intentionnel, naturellement, disparaît. Par exemple dans Ce sont les volets fermés qui m’ont trompé : j'ai cru qu'il n'y avait personne, qu'on peut retourner au passif en disant : J'ai été trompé par les volets fermés : j'ai cru qu'il n'y avait personne, les volets n'ont évidemment aucune intention. Entre la tromperie intentionnelle et la cause de l'erreur, la construction pronominale présente une interprétation qu'il ne faudrait pas confondre avec le passif simple. J'ai été trompé suppose une source d'erreur, intentionnelle s'il s'agit d'êtres humains, simplement causale s'il s'agit d'une situation. Au contraire, je me suis trompé suppose que l'erreur n'a pas été provoquée par un facteur externe. C'est une façon de dire que rien ni personne ne m’a trompé : je me suis trompé tout seul. De ce point de vue, il y a bien convergence avec le nom erreur, qui repose sur un prédicat qui ne prend en compte que celui qui commet l'erreur. En somme la diathèse pronominale du verbe tromper permet d'ajuster son sens avec celui du nom erreur, malgré la divergence des signifiants.

Une autre différence entre les emplois transitifs et l'emploi pronominal mérite d'être soulignée. Très souvent, le verbe tromper transitif, à l'actif comme au passif renvoie au fait d'être infidèle dans une relation de couple, et donc par ailleurs au fait d'abuser quelqu'un. Il y a là un lien sous-jacent à l'étymologie, le coup de trompe ou de trompette étant censé suggérer une forme de moquerie, représentation à rapprocher peut-être du ricanement. En revanche, dans les emplois où l'intention malveillante est absente, donc en particulier dans l'emploi pronominal, la moquerie disparaît ipso facto. Du coup l'écart avec le sens intentionnel se creuse davantage : en se trompant on ne se moque pas de soi-même, même si, comme on l'a vu, on n'est victime de rien ni de personne. La perte de la moquerie renforce inversement les liens avec le nom erreur qui lui sert de nom d'action soit en tant que processus, soit en tant que résultat. En effet, dans les deux cas le fait de prendre pour faux ce qui est vrai ou l'inverse est involontaire et se trouve donc incompatible avec la moquerie.

Si la diathèse pronominale du verbe tromper implique une rupture avec l'étymologie, on peut s'interroger sur ce qu'il en est en ce qui concerne l'erreur. On a vu que le nom erreur en français contemporain avait pris ses distances avec le verbe errer, d'une part en ce que ce verbe tendait à perdre son sens de " commettre une erreur » et d'autre part du fait que le nom erreur avait perdu 
le sens d'" aller à l'aventure ", que le nom errance avait pris en exclusivité. Et pourtant la représentation récurrente de l'erreur fait volontiers intervenir l'idée d'un cheminement : on dit couramment qu'on a pris une mauvaise direction, qu'on s'écarte ou qu'on s'éloigne de la vérité. Autrement dit, l'image d'origine perdure alors même que la polysémie s'est réduite. Cela vaut évidemment pour le verbe se tromper autant que pour le nom erreur.

En revanche, les compléments du nom erreur et du verbe se tromper divergent sur le plan de leur interprétation. Faire une erreur de calcul ne signifie pas qu'on s'est trompé de calcul, mais qu'on s'est trompé dans ses calculs. De même se tromper de rue ne signifie pas qu'on a fait une erreur de rue (dont on ne sait pas très bien ce que cela pourrait vouloir dire !). Dans le cas du nom erreur, le complément en de renvoie au domaine concerné, précisément circonscrit par le complément en dans avec le verbe se tromper. Quant au complément en de du verbe, il repose sur un choix virtuel entre deux ou plusieurs possibilités, situation qui n'est pas retenue spécialement dans la construction du nom erreur. Alors que le verbe oriente vers un choix, le nom réfère plutôt à un parcours.

On notera enfin que le nom erreur peut être introduit par le verbe commettre, ce qui l'oriente de façon un peu surprenante vers un aspect moral, puisque ce que l'on commet relève en principe d'une faute.

En résumé, si le nom erreur et le verbe se tromper fonctionnent en bonne partie en parallèle et se correspondent, l'absence de verbe apparenté d'un côté et l'absence de nom morphologiquement relié de l'autre y trouvant une convergence naturelle, des différences subsistent et contribuent à éloigner partiellement les deux termes. On trouve dans les deux l'idée du caractère involontaire et inconscient ainsi que l'idée d'un cheminement. Mais en même temps, l'erreur retrouve en partie son étymologie en renvoyant à un parcours, tandis que le fait de se tromper relève d'un choix. Et alors que se tromper ne semble pas impliquer de faute morale, l'erreur en comporte des traces.

La plupart des ouvrages que j'ai consultés à propos de l'erreur partent d'une vision plutôt négative de l'erreur ${ }^{5}$. Il s'agit le plus souvent de classer les erreurs et de trouver le moyen de s'en prémunir. Il y a au moins quelques exceptions récentes, comme le petit livre de Laurent Degos, qui évoque le point de vue d'un chercheur dans le domaine des sciences médicales ${ }^{6}$, ou Edgar Morin dans son ouvrage très stimulant sur ses conceptions de l'enseignement et de l'édu-

5 Voir par exemple Olivier Giroud-Fliegner, Tout le monde peut se tromper, Essai sur l'erreur, Paris, Seuil, 2001 ; Luc de Brabandere et Anne Mikolajczak, Petite philosophie de nos erreurs quotidiennes, Paris, Eyrolles, 2009 ; Kathryn Schulz, Cherchez l'erreur! Pourquoi il est profitable d'avoir tort, Paris, Flammarion, 2012 ; Jean Robillard, Petit traité de l'erreur, Montréal, Liber, 2012.

6 Laurent Degos, Éloge de l'erreur, Paris, éd. du Pommier, 2013. 
cation qui comporte quelques pages originales sur "l'erreur de sous-estimer l'erreur ", où est évoqué en particulier l'ouvrage précédent ${ }^{7}$. Mon objectif ici n'est pas de faire l'éloge de l'erreur au nom de l'obscurantisme mais il s'agit de montrer, à partir des éléments que la description sémantique précédente a révélés, ce que l'erreur peut apporter à la recherche dans le domaine de ce qu'on appelle les sciences humaines, au sens le plus large.

Une recherche c'est d'abord un parcours, ce qui la rapproche de l'erreur, dont on a vu qu'elle reposait aussi sur un parcours. La notion de parcours suppose elle-même que l'on se déplace d'un endroit à un autre dans l'intention d'atteindre un objectif. Plusieurs obstacles s'y opposent néanmoins dans le cas de la recherche. Le premier consiste dans l'ignorance du chemin. Si on le connaissait déjà, il n'y aurait plus de recherche. Mais il ne s'agit même pas d'un chemin tout tracé, sinon on ne sortirait pas de ce qu'on appelle les sentiers battus. Il faut donc se frayer un chemin, ce qui implique une forme d'errance. Il y a alors précisément dans toute recherche un risque d'erreur permanent, qui est en même temps la garantie que l'on ne répète pas les mêmes trajets que ceux que d'autres ont déjà empruntés. On sait que la connaissance de ce que d'autres ont fait ou écrit permet de se protéger contre la simple répétition du déjà dit et du déjà vu, que la recherche prétend dépasser (sans forcément les contredire). L'errance c'est ne pas savoir où l'on va ni par où il faut passer si on croit le savoir. Autrement dit l'errance ouvre l'angoissant espace de l'inconnu. Il est clair en même temps que c'est précisément l'inconnu qui constitue le moteur de la recherche pour le chercheur, une sorte de goût de l'aventure. Cela conduit alors sans relâche à un jeu d'espoirs et de déceptions, les chemins sans issues étant beaucoup plus fréquents que les voies de la découverte ${ }^{8}$.

Évidemment l'aventure de la recherche ne part pas de rien. On connait plus ou moins bien les travaux des prédécesseurs. Plus ou moins bien, car lire ce n'est pas forcément comprendre. En même temps, si l'on doit avoir tout lu et tout compris avant de se lancer dans sa propre recherche, on risque de ne jamais commencer à chercher par soi-même. Un autre moyen pour échapper au hasard improductif de l'errance consiste à s'aider d'une méthode, souvent déjà éprouvée sur d'autres sujets ou des sujets voisins, par soi ou par d'autres. La méthode rassure car elle assimile l'inconnu à du connu. Littéralement et étymologiquement elle accompagne le chercheur et le soutient. À nouveau en effet c'est la métaphore du chemin qui définit étymologiquement la recherche, hodos en grec c'est le chemin, la voie, et methodos ${ }^{9}$ le chemin qui poursuit

7 Edgar Morin, Enseigner à vivre, op. cit., p. 73-75.

8 On pourra se reporter sur ce sujet aux propos du physicien Jean-Marc Lévy-Leblond, dans l'émission «À voix nue » du 15 janvier 2015 sur France-Culture : http://www.franceculture.fr/ personne-jean-marc-1\%C3\%A9vy-leblond.html.

9 Le préfixe meta dans met-hodos n'a pas le sens comitatif, courant ailleurs, de " avec ", mais le sens spatio-temporel de «à la (pour)suite de ». 
un objet, en somme c'est la recherche même. Et on ne s'étonnera pas que le verbe grec methodeuein signifie « suivre méthodiquement, rechercher avec méthode » et finalement " tromper »! Décidément la recherche est la sœur de l'erreur. Malheureusement, on sait que la méthode se transforme rapidement en routine, soulageant merveilleusement le chercheur de la peur de l'inconnu.

Cela n'empêche pas qu'il faut bien suivre un chemin, et donc assumer le risque de l'erreur. Car un chemin ne se révèle pas immédiatement sans issue. De même qu'il ne se révèle pas davantage immédiatement fructueux. C'est un des paradoxes de l'erreur. Toutes les erreurs ne sont pas objectivement telles: ce qui semble une erreur peut ne pas en être une au bout du chemin. C'est prendre en défaut le proverbe Errare humanum est, perseverare diabolicum. En ce sens, l'erreur n'est pas simplement un défaut qu'il faut soigneusement éviter, ce qui peut rendre la recherche désespérante, c'est aussi un moyen de se décentrer par rapport au carcan des habitudes de pensée. Et cela vaut aussi pour le rapport à autrui, c'est-à-dire la lecture des travaux des autres. Lire de travers n'est pas toujours mal lire.

Ce caractère relatif de l'erreur me parait plus spécialement notable dans le domaine des sciences humaines. Si se tromper c'est prendre ce qui est faux pour vrai et inversement, cela suppose un point de vue suffisamment objectif pour définir sans aucun doute le vrai et le faux. Or la nature des démonstrations en sciences humaines permet rarement de conduire ne serait-ce qu'à l'unanimité. Mais plus largement, il suffit de se référer à l'histoire des sciences pour constater que toute vérité a une dimension historique, tant dans sa genèse que dans son devenir. Un changement de paradigme suppose précisément que le socle de la vérité soit ébranlé. Ce qui, d'un certain point de vue, est une erreur peut, d'un autre point de vue, constituer une avancée de la connaissance. Or il se trouve que les sciences humaines partagent cette propriété d'être particulièrement plurithéoriques, en ce sens qu'elles se caractérisent par une grande diversité de points de vue possibles. Les vérités des uns y sont donc facilement les erreurs des autres. Cela rend l'erreur d'autant plus respectable, dans le sens où on a intérêt à la regarder deux fois, une erreur pouvant être la face cachée d'une vérité.

On a vu que se tromper était le résultat d'un choix. Cela implique que les termes du choix possédaient une ressemblance suffisante pour induire en erreur. On touche là à ce qui peut être instructif dans l'erreur : le fait de ne pas avoir su faire le bon choix peut amener à s'interroger sur des différences que l'on n'a pas faites. En ce sens l'erreur est acceptée en tant que telle, mais le fait de l'accepter permet de la dépasser et donc de progresser dans la compréhension de l'objet de recherche. La démarche revient alors à lever provisoirement la censure qui tend à faire de l'erreur pour le chercheur une faute. De même qu'on tolèrera l'erreur comme errance, acceptant le risque de l'aventure pour aller affronter nos certitudes, de même la compréhension de nos erreurs 
permet non seulement de mieux les éviter mais aussi de mieux progresser dans nos recherches. De ce point de vue, l'erreur se présente à nous un peu comme un effet du refoulement : nous avons vu que l'une des propriétés essentielles de l'erreur est d'être involontaire. Le surmoi du vrai ne suffit pas à nous affranchir de l'erreur, il nous faut en quelque sorte tenter de l'analyser. Il ne s'agit donc pas de reconnaitre nos erreurs, ce qui renforcerait l'interdit qu'elles font peser sur nos recherches, il faut au contraire les accepter comme des effets de la dynamique de notre recherche. L’objectif est alors de faire émerger au niveau de la conscience ce qui nous pousse à nous tromper et donc à manquer la cible.

Si l'on revient à la notion d'errance, on notera une propriété qui peut contribuer à faire apparaitre une autre valeur de l'erreur. Il s'agit du fait que l'errance constitue un déplacement aléatoire. Or il se trouve que le travail de recherche, quel que soit son degré d'organisation et de respect des protocoles en vigueur, comporte une part non négligeable de rencontres imprévisibles. Ce rôle du hasard correspond précisément au concept de sérendipité inventé par Horace Walpole à partir du vieux nom de l'île de Sri Lanka, Serendip, d'origine persane. Au hasard s'ajoute, dans la sérendipité, le caractère inattendu, qui correspond au caractère non contrôlé et involontaire de l'erreur. C'est en quelque sorte une erreur fertile. C'est aussi, d'une certaine façon la condamnation des programmes de recherche, dans la mesure du moins où on les considère comme susceptibles de maitriser parfaitement leur déroulement et leur capacité à atteindre leurs objectifs. Dans le cas contraire, cela reviendrait à dire que la recherche est d'autant plus efficace qu'elle repose sur une absence totale d'imprévisibilité. Ce qui est en cause finalement c'est le degré de liberté que doit absolument conserver le chercheur pour alimenter son désir de recherche ainsi que sa capacité de découverte. Mais cette dynamique est en même temps celle de l'erreur, qu'elle soit positive ou négative. Erreur quotidienne qui doit être constamment corrigée, mais aussi erreur heuristique, grâce à laquelle l'inconnu nous est un peu moins inconnu.

Au terme de ce bref parcours, j'espère avoir montré que les représentations associées au nom erreur et au verbe se tromper permettent de se faire une idée en partie positive du rôle de l'erreur dans le travail de recherche. Nous avons vu que la métaphore la plus importante était celle du chemin, ce qui suppose un espace à parcourir en terrain inconnu, avec un point de départ et un objectif (une destination) à atteindre. Or à tout moment du trajet, ce qu'on appelle l'erreur peut se manifester tant du côté de ce qu'on peut appeler le socle même de nos connaissances que sur les directions à prendre ou les objectifs à atteindre. L'indétermination de ces composantes du chemin du chercheur signifie qu'à chaque instant de son parcours la recherche implique une forme d'errance et la nécessité d'un choix. L'erreur se trouve ainsi intimement et intrinsèquement associée à la recherche. Dès lors ce n'est pas l'évite- 
ment systématique de l'erreur qu'il faut viser mais plutôt l'analyse de celles que le mouvement même de la recherche nous amène à " commettre ". C'est donc de l'innocente liberté de l'erreur dont nous avons besoin et non de la prison des méthodes.

Frédéric Lambert

UMR 5263 CLLE-ERSSàB

Université Bordeaux Montaigne

frederic.lambert@u-bordeaux-montaigne.fr

\section{Résumé}

Les caprices de l'histoire du français ont fait que le nom erreur a pour verbe correspondant se tromper. À partir d'une étude de la signification de ces deux termes, de leur histoire et de leur domaine conceptuel, quelques propriétés apparaissent, qui confirment une grande convergence entre les deux termes. Le chemin, l'errance et le choix sont les principales. Ces représentations permettent de concevoir l'erreur à la fois comme une nécessité et une richesse pour la recherche.

\section{Mots-clés}

Erreur, métaphore, recherche, errance, choix.

\section{Abstract}

Due to the whims of the history of the French language, to the noun erreur corresponds the verb se tromper. Starting from a study of the semantics of these two items, of their history and of their conceptual domain, some features appear, which confirm a mainly common ground between the two items. The main ones are the notions of path, wandering and choice. Through these images, error can be conceived both as a necessity and as a wealth for scientific research.

\section{Keywords}

Error, metaphor, research, wandering, choice. 